\title{
Uniform distribution of generalized polynomials of the product type
}

\author{
by \\ IngER Johanne HÅland (Grimstad)
}

1. Introduction. H. Weyl showed in [6] that if $p(x)$ is a real-valued polynomial with at least one irrational coefficient other than the constant term, then the sequence $p(n), n=1,2, \ldots$, is uniformly distributed $(\bmod 1)$. Generalized polynomials form a natural family of functions which are obtained from the polynomials by the use of the greatest integer function [.], addition and multiplication. For example, $[\alpha x] \beta x^{2}$ and $[\alpha x][\beta x] \gamma$ are generalized polynomials. It was shown in [3] that if the coefficients of a generalized polynomial $q(x)$ are sufficiently independent, then the sequence $q(n), n=1,2, \ldots$, is uniformly distributed $(\bmod 1)$. In this paper we show the following result.

TheOREM 1.1. For any $\alpha_{1}, \ldots, \alpha_{k} \in \mathbb{R} \backslash\{0\}, k \geq 3$, and for any irrational $\gamma$ the sequence

$$
q(n)=\left[\alpha_{1} n\right]\left[\alpha_{2} n\right] \ldots\left[\alpha_{k} n\right] \gamma, \quad n=1,2, \ldots,
$$

is uniformly distributed $(\bmod 1)$.

Note that the same is not true for $k=1$ and $k=2$. When $k=1$, the identity

$$
[\alpha n] \beta \equiv \alpha \beta n-\{\alpha n\} \beta(\bmod 1)
$$

implies that the sequence $[\alpha n] \beta$ is uniformly distributed $(\bmod 1)$ if and only if $\beta$ is rationally independent of $1,1 / \alpha$ [4, Theorem 5.1.8]. In the case $k=2$ we have the following proposition [3, Proposition 5.3].

Proposition 1.2. The generalized polynomial $[\alpha n][\beta n] \gamma$ is uniformly distributed $(\bmod 1)$ if and only if one of the following conditions hold:

(i) $\alpha / \beta \neq \sqrt{c}$ for all $c \in \mathbb{Q}^{+}$and $\gamma$ is irrational.

(ii) $\alpha / \beta=\sqrt{c}$ for some $c \in \mathbb{Q}^{+}$and $\gamma$ is rationally independent of $1, \sqrt{c}$.

This result is part of the author's Ph.D. thesis done under the direction of Prof. V. Bergelson at Ohio State University. 
The identity

$$
[\beta \sqrt{c} n][\beta n] \sqrt{c} \equiv-\frac{1}{2}(\{\beta \sqrt{c} n\}-\sqrt{c}\{\beta n\})^{2}(\bmod 1)
$$

is the reason why the sequence $[\beta \sqrt{c} n][\beta n] \sqrt{c}$ fails to be uniformly distributed $(\bmod 1)$. Note, however, that by Theorem 1.1 , there cannot be any such identities for $k \geq 3$.

Our method is, as in [3], based on the following useful theorem by van der Corput [2].

Theorem 1.3 (van der Corput's difference theorem). Let $x_{n}, n=1$, $2, \ldots$, be a real-valued sequence. If there is some $h_{0} \in \mathbb{N}$ such that for all integers $h \geq h_{0}$ the sequence $x_{n+h}-x_{n}, n=1,2, \ldots$, is uniformly distributed $(\bmod 1)$, then $x_{n}$ is also uniformly distributed $(\bmod 1)$.

If $p(n)$ is a usual polynomial then $p^{h}(n)=p(n+h)-p(n)$ is a new polynomial of degree $\operatorname{deg}(p)-1$. Therefore, Weyl's theorem for polynomials with irrational leading coefficients follows easily by van der Corput's difference theorem and induction since the sequence $\alpha n+\beta$ is uniformly distributed $(\bmod 1)$ if and only if $\alpha$ is irrational. See for example [4] for the complete proof of Weyl's theorem. The same idea will be used to prove Theorem 1.1. However, the proof is more complicated because of the brackets in the expressions of $q(n)$.

The special case $[\alpha n]^{k} \gamma$ of Theorem 1.1 is proved in $[5$, Cor. 3.5 and the following Example] by the use of spectral theory. Uniform distribution of $[\alpha n]^{k} \gamma$, where $1, \alpha, \alpha \gamma$ are rationally independent, can also be proved by ergodic theoretical methods. This follows from a modified version of [1].

2. The van der Corput method. Denote by $[r]$ the greatest integer less than or equal to the real number $r$, and by $\{r\}$ the fractional part of $r$, so that $r=[r]+\{r\}$.

Definition 2.1. A real-valued sequence $x(n), n=1,2,3, \ldots$, is uniformly distributed $(\bmod 1)$ if for any real numbers $0 \leq a<b \leq 1$,

$$
\lim _{N \rightarrow \infty} \frac{1}{N} \operatorname{card}(\{1 \leq n \leq N \mid\{x(n)\} \in[a, b)\})=b-a .
$$

Similarly, uniform distribution $(\bmod 1)$ of sequences in $\mathbb{R}^{l}, l>1$, is defined. We will need the following theorem.

Theorem $2.1([4])$. Let $\underline{x}(n)=\left(x_{1}(n), \ldots, x_{l}(n)\right)$ be a sequence in $\mathbb{R}^{l}$. Then the following statements are equivalent:

(i) $\underline{x}(n)$ is uniformly distributed $(\bmod 1)$ in $\mathbb{R}^{l}$.

(ii) $\sum_{i=1}^{l} k_{i} x_{i}(n)$ is uniformly distributed $(\bmod 1)$ in $\mathbb{R}$ for all l-tuples $\left(k_{1}, \ldots, k_{l}\right) \neq(0, \ldots, 0)$ of integers. 
(iii) For every Riemann integrable function $f$ on $[0,1]^{l}$,

$$
\lim _{N \rightarrow \infty} \frac{1}{N} \sum_{n=0}^{N-1} f(\{\underline{x}(n)\})=\int_{0}^{1} \ldots \int_{0}^{1} f(\mathbf{x}) d x_{1} \ldots d x_{l} .
$$

Definition 2.2. Define the degree of a generalized polynomial

$$
q(n)=\sum_{i=1}^{l} \prod_{j=1}^{k_{i}}\left[\alpha_{i j} n+\beta_{i j}\right] \gamma_{i}
$$

for which

$$
\prod_{j=1}^{k_{i}}\left[\alpha_{i j} n+\beta_{i j}\right] \neq \prod_{j=1}^{k_{r}}\left[\alpha_{r j} n+\beta_{r j}\right] \quad \text { if } i \neq r
$$

to be

$$
\operatorname{deg}(q)=\max \left\{k_{i} \mid 1 \leq i \leq l\right\} .
$$

This way the degree of $q(n)=\left[\alpha_{1} n\right] \ldots\left[\alpha_{k} n\right] \gamma$ is $k$. Theorem 1.1 will be proved by induction on $\operatorname{deg}(q)$. By van der Corput's difference theorem, it suffices to prove that $q^{h}(n)=q(n+h)-q(n)$ is uniformly distributed ( $\bmod$ 1 ) for all but finitely many $h$ 's. However, the degree of $q^{h}(n)$ is the same as that of $q(n)$ and not lower as in the polynomial case. Therefore we need to find a new generalized polynomial $V_{h} q(n)$ from $q^{h}(n)$ which has degree $\operatorname{deg}(q)-1$ and which can be used instead of $q^{h}(n)$. For now, let

$$
q(n)=\sum_{i=1}^{l}\left(\prod_{j=1}^{k_{i}}\left[\alpha_{i j} n\right]\right) \gamma_{i}
$$

Since

$$
[\alpha(n+h)]=[\alpha n]+[\alpha h]+1_{A}(\alpha n, \alpha h),
$$

where $A=\left\{(x, y) \in[0,1)^{2} \mid x+y \geq 1\right\}$ such that

$$
1_{A}(\alpha n, \alpha h)= \begin{cases}1 & \text { if }\{\alpha n\}+\{\alpha h\} \geq 1, \\ 0 & \text { if }\{\alpha n\}+\{\alpha h\}<1,\end{cases}
$$

we have

$$
q^{h}(n)=\sum_{i=1}^{l} \sum_{r=1}^{k_{i}}\left[\alpha_{i r} h\right]\left(\prod_{j \neq r}\left[\alpha_{i j} n\right]\right) \gamma_{i}+s(n)+\sum_{i} 1_{C_{i}}(*) t_{i}(n)
$$

where $s(n)$ and $t_{i}(n)$ are all of the form

$$
\sum_{i=1}^{k_{0}} a_{i}(h)\left(\prod_{j=1}^{l_{i}}\left[\alpha_{i r_{j}} n\right]\right) \gamma_{i}, \quad l_{i}<k_{i}-1, a_{i}(h) \in \mathbb{Q} .
$$


The components of the argument $(*)$ of the indicator function $1_{C_{i}}$, where $C_{i} \subset[0,1)^{s_{i}}$ for some $s_{i}$, are constants and linear polynomials whose coefficients can be written as linear combinations over $\mathbb{Q}$ of some rationally independent numbers $1, \beta_{1}, \ldots, \beta_{l}$. Let

$$
V_{h} q(n)=\sum_{i=1}^{l} \sum_{r=1}^{k_{i}}\left[\alpha_{i r} h\right]\left(\prod_{j \neq r}\left[\alpha_{i j} n\right]\right) \gamma_{i} .
$$

To prove that $q^{h}(n)$ is uniformly distributed $(\bmod 1)$ it is enough by Theorem 2.1 to prove that

$$
\left(V_{h} q(n)+s(n)+\sum_{i} \varepsilon_{i} t_{i}(n), \beta_{1} n, \ldots, \beta_{l} n\right)
$$

is uniformly distributed $(\bmod 1)$ in $\mathbb{R}^{l+1}$ for any $\varepsilon_{i} \in\{0,1\}$, or equivalently, that

$$
q^{\left(b_{i}, \varepsilon_{i}, h\right)}(n)=V_{h} q(n)+s(n)+\sum_{i} \varepsilon_{i} t_{i}(n)+\sum_{i=1}^{l} b_{i} \beta_{i} n
$$

is uniformly distributed $(\bmod 1)$ for any $\varepsilon_{i} \in\{0,1\}$ and any $b_{i} \in \mathbb{Q}$. Therefore, $q(n)$ is uniformly distributed $(\bmod 1)$ by van der Corput's difference theorem if $q^{\left(b_{i}, \varepsilon_{i}, h\right)}(n)$ is uniformly distributed $(\bmod 1)$ for any $b_{i}, \varepsilon_{i}, h \geq h_{0}$ for some $h_{0} \in \mathbb{N}$. Note that the degree of $q^{\left(b_{i}, \varepsilon_{i}, h\right)}(n)$ is $\operatorname{deg}(q)-1$. By repeating this process $\operatorname{deg}(q)-2$ times we obtain generalized polynomials of degree two whose terms of degree two are all coming from $V_{h} q(n)$. It follows from [3] that if a generalized polynomial $q_{1}(n)$ is uniformly distributed (mod $1)$ and $\operatorname{deg}\left(q_{1}\right)=2$, then $q_{1}(n)+q_{0}(n)$ is also uniformly distributed $(\bmod 1)$ for any generalized polynomial $q_{0}(n)$ of degree one. Therefore, the following proposition applies to these new generalized polynomials of degree two when the identity

$$
[a] b+[b] a=a b+[a][b]-\{a\}\{b\}
$$

is used.

Proposition $2.2([3])$. Let $1, \alpha_{1}, \ldots, \alpha_{k}$ be rationally independent. Then

$$
q(n)=\sum_{i=1}^{k}\left[\alpha_{i} n\right] \beta_{i} n+\alpha_{0} n^{2}
$$

is uniformly distributed $(\bmod 1)$ if and only if one of the following conditions holds:

(i) There exists $i$ such that $\beta_{i}$ is rationally independent of $1, \alpha_{1}, \ldots, \alpha_{k}$.

(ii) $\beta_{i}=a_{i 0}+\sum_{j=1}^{k} a_{i j} \alpha_{j}, a_{i j} \in \mathbb{Q}, i=1, \ldots, k$, and there exist $i, j$ such that $a_{i j} \neq a_{j i}$. 

and

(iii) $\beta_{i}=a_{i 0}+\sum_{j=1}^{k} a_{i j} \alpha_{j}, a_{i j} \in \mathbb{Q}, i=1, \ldots, k, a_{i j}=a_{j i}$ for all $i, j$

$$
\sum_{i=1}^{k} \sum_{j=1}^{i-1} a_{i j} \alpha_{i} \alpha_{j}+\frac{1}{2} \sum_{i=1}^{k} a_{i i} \alpha_{i}^{2}+\alpha_{0} \notin \mathbb{Q} .
$$

If $q(n)$ can be shown to be uniformly distributed $(\bmod 1)$ by this process we will say that $q(n)$ is uniformly distributed $(\bmod 1)$ by the van der Corput method.

Not at i o n. We will say that $q_{1}(n) \sim q_{2}(n)$ if there exist a Riemann integrable periodic mod 1 function $g$ on $\mathbb{R}^{m}$, generalized polynomials $v_{0}(n), \ldots$, $v_{l}(n)$, each of the form (1) and so that $\operatorname{deg}\left(v_{i}\right)<\min \left\{\operatorname{deg}\left(q_{1}\right), \operatorname{deg}\left(q_{2}\right)\right\}$, $i=0, \ldots, k$, and rationally independent numbers $1, \beta_{1}, \ldots, \beta_{m}$ such that

$$
q_{1}(n)-q_{2}(n)=v_{0}(n)+\sum_{i=1}^{l} 1_{C_{i}}(*) v_{i}(n)+g\left(\beta_{1} n, \ldots, \beta_{m} n\right)(\bmod 1)
$$

where the components of the arguments $(*)$ are linear combinations of constants and $\beta_{i} n$ 's.

Note that $q^{h}(n) \sim V_{h} q(n)$ and that we have the following lemma.

LEMMA 2.3. If $q_{1}(n) \sim q_{2}(n)$ then $q_{1}(n)$ is uniformly distributed $(\bmod 1)$ by the van der Corput method if and only if $q_{2}(n)$ is uniformly distributed $(\bmod 1)$ by the van der Corput method.

Lemma 2.4 ([3]). Let $\lambda_{0}, \lambda_{1}, \ldots, \lambda_{k}$ be rationally independent and let $R \subset \mathbb{R}$. Then $\sum_{i=0}^{k}\left[\lambda_{i} h\right] \theta_{i}$ is rationally independent of $R$ for all but finitely many $h$ if and only if there exists $j, 0 \leq j \leq k$, such that $\theta_{j}$ is rationally independent of $R$.

LEMMA 2.5. If $\lambda_{1}, \ldots, \lambda_{l}$ are rationally independent and $q_{h}(n)=$ $\sum_{i=1}^{l}\left[\lambda_{i} h\right] u_{i}(n)$, where each $u_{i}(n)$ is of the form (1) and $\operatorname{deg}\left(u_{i}\right)=\operatorname{deg}\left(q_{h}\right) \geq$ 2 , then $q_{h}(n)$ is uniformly distributed $(\bmod 1)$ for all but finitely many $h$ if some $u_{i}(n)$ is uniformly distributed $(\bmod 1)$ by the van der Corput method.

Rem ark. This may fail if $\operatorname{deg}\left(q_{h}\right)=1$. Indeed, let $1, \lambda_{1}, \lambda_{2}$ and $1, \alpha_{1}, \alpha_{2}$ be rationally independent, and let

$$
\begin{aligned}
q_{h}(n) & =\left[\lambda_{1} h\right]\left[\alpha_{1} n\right] \beta_{1}+\left[\lambda_{2} h\right]\left[\alpha_{2} n\right] \beta_{2} \\
& =\left[\lambda_{1} h\right] \alpha_{1} \beta_{1} n+\left[\lambda_{2} h\right] \alpha_{2} \beta_{2} n-\left[\lambda_{1} h\right]\left\{\alpha_{1} n\right\} \beta_{1}-\left[\lambda_{2} h\right]\left\{\alpha_{2} n\right\} \beta_{2} .
\end{aligned}
$$

If $\alpha_{2}=\alpha_{1} \beta_{1}$ and $\alpha_{1}=\alpha_{2} \beta_{2}$, then both $\left[\alpha_{1} n\right] \beta_{1}$ and $\left[\alpha_{2} n\right] \beta_{2}$ are uniformly distributed $(\bmod 1)$, but

$$
q_{h}(n)=\left(\left[\lambda_{1} h\right]-\left[\lambda_{2} h\right] \beta_{2}\right)\left\{\alpha_{2} n\right\}+\left(\left[\lambda_{2} h\right]-\left[\lambda_{1} h\right] \beta_{1}\right)\left\{\alpha_{1} n\right\}
$$

is not uniformly distributed $(\bmod 1)$. 
Proof of Lemma 2.5. Suppose $\operatorname{deg}\left(q_{h}\right)=2$. Since by $(2)$,

$$
\left[\alpha_{1} n\right]\left[\alpha_{2} n\right] \gamma \sim\left[\alpha_{1} n\right] \alpha_{2} \gamma n+\left[\alpha_{2} n\right] \alpha_{1} \gamma n-\alpha_{1} \alpha_{2} \gamma n^{2}
$$

we may write each $u_{i}(n)$ in the form $\sum_{j}\left[\alpha_{j} n\right] \beta_{j} n+\alpha_{0} n^{2}$. Furthermore, by using the relations $[\alpha n] \beta n \sim \alpha \beta n^{2}-[\beta n] \alpha n$ and $[\alpha n] \alpha n \sim \frac{1}{2} \alpha^{2} n^{2}$ we can reduce any $u_{i}(n)$ which is not uniformly distributed $(\bmod 1)$ to a polynomial $a n^{2}, a \in \mathbb{Q}$. Therefore we may assume that each $u_{i}(n)$ is uniformly distributed $(\bmod 1)$. Also, if not all the $u_{i}(n)$ 's are polynomials, let $k \geq 1$ be the smallest integer such that there exist rationally independent numbers $1, \alpha_{1}, \ldots, \alpha_{k}$ with

$$
u_{i}(n)=\sum_{j=1}^{k}\left[\alpha_{j} n\right] \beta_{i j} n+\beta_{i 0} n^{2},
$$

for some $\beta_{i j} \in \mathbb{R}$. Then there exists some $\beta_{i j}$ rationally independent of $1, \alpha_{1}, \ldots, \alpha_{k}$. Hence, by Lemma 2.4 , there exists at least one $j$ so that $\sum_{i=1}^{l}\left[\lambda_{i} h\right] \beta_{i j}$ is rationally independent of $1, \alpha_{1}, \ldots, \alpha_{k}$ for all but finitely many $h$. Now,

$$
\begin{aligned}
q_{h}(n) & =\sum_{i=1}^{l}\left[\lambda_{i} h\right]\left(\sum_{j=1}^{k}\left[\alpha_{j} n\right] \beta_{i j} n+\beta_{i 0} n^{2}\right) \\
& =\sum_{j=1}^{k}\left[\alpha_{j} n\right]\left(\sum_{i=1}^{l}\left[\lambda_{i} h\right] \beta_{i j}\right) n+\sum_{i=1}^{l}\left[\lambda_{i} h\right] \beta_{i 0} n^{2} .
\end{aligned}
$$

So if $q_{h}(n)$ is not a polynomial, it follows from Proposition 2.2 that $q_{h}(n)$ is uniformly distributed $(\bmod 1)$ for all but finitely many $h$. If $q_{h}(n)$ is a polynomial, then the coefficient $\sum_{i=1}^{l}\left[\lambda_{i} h\right] \beta_{i 0}$ is irrational for all but finitely many $h$ by Lemma 2.4. This proves the degree two case.

We prove the general statement by induction on $\operatorname{deg}\left(q_{h}\right)$. Assume it is true if $\operatorname{deg}\left(q_{h}\right)<d$ and let $\operatorname{deg}\left(q_{h}\right)=d>2$. We have

$$
q_{h}^{k}(n)=q_{h}(n+k)-q_{h}(n)=\sum_{i=1}^{l}\left[\lambda_{i} h\right] u_{i}^{k}(n) \sim \sum_{i=1}^{l}\left[\lambda_{i} h\right] V_{k} u_{i}(n)
$$

where $\operatorname{deg}\left(V_{k} u_{i}\right)=\operatorname{deg}\left(u_{i}\right)-1$. Since at least one $u_{i}(n)$ is uniformly distributed $(\bmod 1)$ by the van der Corput method, there is some $u_{i}^{k}(n)$ and hence $V_{k} u_{i}(n)$ by Lemma 2.3 , which is uniformly distributed $(\bmod 1)$ for all but finitely many $k$. So by the induction hypothesis $q_{h}^{k}(n)$ is uniformly distributed $(\bmod 1)$ for all but finitely many $k$ and $h$. Hence, by van der Corput's difference theorem, $q_{h}(n)$ is uniformly distributed $(\bmod 1)$ for all but finitely many $h$. 
Re mark. When the coefficients $\alpha_{1}, \ldots, \alpha_{k}$ of a generalized polynomial $q(n)$ are rationally dependent, say

$$
\alpha_{k}=\sum_{i=1}^{k-1} \frac{r_{i}}{s_{i}} \alpha_{i}, \quad r_{i}, s_{i} \in \mathbb{Z},
$$

we will in the proofs assume that $s_{i}=1, i=1, \ldots, k-1$. Then the general case, when $s_{i} \neq 1$, follows by writing $n=m d+b, 0 \leq b<d$, in the expression for $q(n)$, where $d$ is the least common multiple of the $s_{i}$ 's. See the proof of [3, Prop. 5.2] for more details.

\section{Some preliminary results}

Proposition 3.1. Let $\lambda_{1}, \lambda_{2}$ be rationally independent numbers, $\gamma$ an irrational number and $b_{0}, b_{1}, b_{2} \in \mathbb{Q}$. A generalized polynomial

$$
Q(n)=b_{0}\left[\lambda_{1} n\right]^{2} \gamma+b_{1}\left[\lambda_{1} n\right]\left[\lambda_{2} n\right] \gamma+b_{2}\left[\lambda_{2} n\right]^{2} \gamma
$$

is uniformly distributed $(\bmod 1)$ unless there exist a, $c, k_{1}, k_{0} \in \mathbb{Q}, c>0$, and $b \in\{+1,-1\}$ such that $\lambda_{2} / \lambda_{1}=a+b \sqrt{c}, \gamma=k_{0}+k_{1} \sqrt{c}$ and

$$
b_{0}+b_{1} a+b_{2}\left(a^{2}-c\right)=0 .
$$

Pro of. By the identity (2), we have

$$
\begin{aligned}
Q(n) \sim & 2 b_{0}\left[\lambda_{1} n\right] \lambda_{1} \gamma n-b_{0} \lambda_{1}^{2} \gamma n^{2}+b_{1}\left[\lambda_{1} n\right] \lambda_{2} \gamma n+b_{1}\left[\lambda_{2} n\right] \lambda_{1} \gamma n \\
& -b_{1} \lambda_{1} \lambda_{2} \gamma n^{2}+2 b_{2}\left[\lambda_{2} n\right] \lambda_{2} \gamma n-b_{2} \lambda_{2}^{2} \gamma n^{2} \\
= & {\left[\lambda_{1} n\right]\left(2 b_{0} \lambda_{1} \gamma+b_{1} \lambda_{2} \gamma\right) n+\left[\lambda_{2} n\right]\left(b_{1} \lambda_{1} \gamma+2 b_{2} \lambda_{2} \gamma\right) n } \\
& -\left(b_{0} \lambda_{1}^{2} \gamma+b_{1} \lambda_{1} \lambda_{2} \gamma+b_{2} \lambda_{2}^{2} \gamma\right) n^{2} \\
= & {\left[\lambda_{1} n\right] A n+\left[\lambda_{2} n\right] B n-\frac{1}{2}\left(\lambda_{1} A+\lambda_{2} B\right) n^{2} }
\end{aligned}
$$

where

$$
A=2 b_{0} \lambda_{1} \gamma+b_{1} \lambda_{2} \gamma, \quad B=b_{1} \lambda_{1} \gamma+2 b_{2} \lambda_{2} \gamma
$$

Suppose first that $1, \lambda_{1}, \lambda_{2}$ are rationally independent. Then by Proposition $2.2, Q(n)$ fails to be uniformly distributed $(\bmod 1)$ if and only if there exist $a_{i} \in \mathbb{Q}$ such that $A=a_{0}+a_{1} \lambda_{1}+a_{2} \lambda_{2}, B=a_{4}+a_{2} \lambda_{1}+a_{3} \lambda_{2}$ and $\frac{1}{2} a_{1} \lambda_{1}^{2}+a_{2} \lambda_{2} \lambda_{1}+\frac{1}{2} a_{3} \lambda_{2}^{2}-\frac{1}{2}\left(\lambda_{1} A+\lambda_{2} B\right)=-\frac{1}{2}\left(a_{0} \lambda_{1}+a_{4} \lambda_{2}\right) \in \mathbb{Q}$, in which case $a_{0}=a_{4}=0$. So $Q(n)$ is not uniformly distributed $(\bmod 1)$ if and only if

(4) $A=a_{1} \lambda_{1}+a_{2} \lambda_{2}$ and $B=a_{2} \lambda_{1}+a_{3} \lambda_{2} \quad$ for some $a_{1}, a_{2}, a_{3} \in \mathbb{Q}$.

Now, if $\lambda_{1}=d \in \mathbb{Q}$, then $Q(n) \sim\left[\lambda_{2} n\right] B n-\frac{1}{2}\left(\lambda_{2} B-d A\right) n^{2}$. Therefore, $Q(n)$ is not uniformly distributed $(\bmod 1)$ if and only if there exist $a_{i} \in \mathbb{Q}$ such that $B=a_{2} d+a_{3} \lambda_{2}$ and $a_{3} \lambda_{2}^{2}-\left(\lambda_{2}\left(a_{2} d+a_{3} \lambda_{2}\right)-d A\right)=d A-a_{2} d \lambda_{2} \in \mathbb{Q}$. So also in this case $Q(n)$ is not uniformly distributed $(\bmod 1)$ if and only if $A$ and $B$ satisfy the condition (4). 
Suppose (4) is true and that $\lambda_{2}$ is rationally independent of $1, \lambda_{1}$. Then by (3) and (4) we have

$$
\begin{aligned}
& 2 b_{0} \lambda_{1} \gamma+b_{1} \lambda_{2} \gamma=a_{1} \lambda_{1}+a_{2} \lambda_{2}, \\
& b_{1} \lambda_{1} \gamma+2 b_{2} \lambda_{2} \gamma=a_{2} \lambda_{1}+a_{3} \lambda_{2},
\end{aligned}
$$

which implies that both $\lambda_{1} \gamma$ and $\lambda_{2} \gamma$ are rationally dependent of $\lambda_{1}, \lambda_{2}$, say

$$
\lambda_{1} \gamma=c_{1} \lambda_{1}+c_{2} \lambda_{2}, \quad \lambda_{2} \gamma=d_{1} \lambda_{1}+d_{2} \lambda_{2},
$$

where $c_{1}, c_{2}, d_{1}, d_{2}$ are given by (5). Note that $c_{2} \neq 0, d_{1} \neq 0$. This gives the equation

$$
2 b_{0} c_{2}+b_{1}\left(d_{2}-c_{1}\right)-2 b_{2} d_{1}=0 .
$$

It follows from (6) that

$$
c_{2} \lambda_{2}^{2}+\left(c_{1}-d_{2}\right) \lambda_{1} \lambda_{2}-d_{1} \lambda_{1}^{2}=0 .
$$

So if (8) is possible, then $\lambda_{2}=(a+b \sqrt{c}) \lambda_{1}$, where $a=\left(d_{2}-c_{1}\right) / 2 c_{2}$, $c=a^{2}+d_{1} / c_{2}$ and $b \in\{+1,-1\}$. By dividing (7) by $2 c_{2}$ we have

$$
b_{0}+a b_{1}+\left(a^{2}-c\right) b_{2}=0 \text {. }
$$

So if $\lambda_{2}$ is rationally independent of $1, \lambda_{1}$, then $Q(n)$ fails to be uniformly distributed $(\bmod 1)$ if and only if there exist $a, c \in \mathbb{Q}$ satisfying $(9)$ and such that for some $b \in\{+1,-1\}$, we have $\lambda_{2}=(a+b \sqrt{c}) \lambda_{1}$ and $\gamma=k_{0}+k_{1} \sqrt{c}$ for some $k_{0}, k_{1} \in \mathbb{Q}$.

If $\lambda_{2}$ is rationally dependent of $1, \lambda_{1}$, say $\lambda_{1}=d_{1}+d_{2} \lambda_{2}$ for some $d_{1}, d_{2} \in$ $\mathbb{Q} \backslash\{0\}$, then

$$
\begin{aligned}
Q(n) & \sim b_{0}\left(d_{1} n+d_{2}\left[\lambda_{2} n\right]\right)^{2} \gamma+b_{1}\left(d_{1} n+d_{2}\left[\lambda_{2} n\right]\right)\left[\lambda_{2} n\right] \gamma+b_{2}\left[\lambda_{2} n\right]^{2} \gamma \\
& =b_{0} d_{1}^{2} n^{2} \gamma+\left(b_{1} d_{1}+2 b_{0} d_{1} d_{2}\right)\left[\lambda_{2} n\right] n \gamma+\left(b_{2}+b_{1} d_{2}+b_{0} d_{2}^{2}\right)\left[\lambda_{2} n\right]^{2} \gamma .
\end{aligned}
$$

By the above result, $Q(n)$ is not uniformly distributed $(\bmod 1)$ if and only if there exist $a, c, b, k_{0}, k_{1} \in \mathbb{Q}$ such that $\lambda_{2}=a+b \sqrt{c}, \gamma=k_{0}+k_{1} \sqrt{c}$ and

$$
\begin{aligned}
0 & =b_{0} d_{1}^{2}+d_{1}\left(b_{1}+2 b_{0} d_{2}\right) a+\left(b_{2}+b_{1} d_{2}+b_{0} d_{2}^{2}\right)\left(a^{2}-c\right) \\
& =b_{0}\left(d_{1}^{2}+2 a d_{1} d_{2}+\left(a^{2}-c\right) d_{2}^{2}\right)+b_{1}\left(a d_{1}+\left(a^{2}-c\right) d_{2}\right)+b_{2}\left(a^{2}-c\right) .
\end{aligned}
$$

So

(10) $\quad b_{0}+b_{1} \frac{a d_{1}+\left(a^{2}-c\right) d_{2}}{d_{1}^{2}+2 a d_{1} d_{2}+\left(a^{2}-c\right) d_{2}^{2}}+b_{2} \frac{a^{2}-c}{d_{1}^{2}+2 a d_{1} d_{2}+\left(a^{2}-c\right) d_{2}^{2}}=0$.

Now,

$$
\frac{\lambda_{2}}{\lambda_{1}}=\frac{a+b \sqrt{c}}{d_{1}+d_{2}(a+b \sqrt{c})}=\frac{d_{1} a+\left(a^{2}-c\right) d_{2}+d_{1} b \sqrt{c}}{d_{1}^{2}+2 a d_{1} d_{2}+\left(a^{2}-c\right) d_{2}^{2}} \equiv a^{\prime}+b \sqrt{c^{\prime}} .
$$


Also,

$$
a^{\prime 2}-c^{\prime}=\frac{\left(d_{1} a+\left(a^{2}-c\right) d_{2}\right)^{2}-d_{1}^{2} c}{\left(d_{1}^{2}+2 a d_{1} d_{2}+\left(a^{2}-c\right) d_{2}^{2}\right)^{2}}=\frac{a^{2}-c}{d_{1}^{2}+2 a d_{1} d_{2}+\left(a^{2}-c\right) d_{2}^{2}} .
$$

Hence, equation (10) is $b_{0}+b_{1} a^{\prime}+b_{2}\left(a^{\prime 2}-c^{\prime}\right)=0$, where $\lambda_{2}=\left(a^{\prime}+b \sqrt{c^{\prime}}\right) \lambda_{1}$, $\gamma=k_{0}^{\prime}+k_{1}^{\prime} \sqrt{c^{\prime}}$.

Lemma 3.2. Let

$$
M=\left(\begin{array}{cccccc}
A_{1} & B_{2} & 0 & 0 & \ldots & 0 \\
C_{1} & A_{2} & B_{3} & 0 & \ldots & 0 \\
0 & C_{2} & A_{3} & B_{4} & \ldots & 0 \\
& & & \ddots & & \\
0 & \ldots & 0 & C_{k-3} & A_{k-2} & B_{k-1} \\
0 & \ldots & 0 & 0 & C_{k-2} & A_{k-1}
\end{array}\right)
$$

where $A_{j}, B_{j}, C_{j} \in \mathbb{Q} \backslash\{0\}$ such that $C_{j} B_{j+1}=d A_{j} A_{j+1}, d=\frac{1}{4}\left(1-c / a^{2}\right)$, $c, a \in \mathbb{Q}, c>0, c \neq a^{2}$. Then $\operatorname{det}(M) \neq 0$.

Proof. Define a sequence $c_{j}$ inductively by $c_{0}=c_{1}=1$ and $c_{j}=$ $c_{j-1}-d c_{j-2}$ for $j \geq 2$. We will show that $c_{j}>0$ for all $j=1, \ldots, k-1$, and that $\operatorname{det}(M)=A_{1} \ldots A_{k-1} c_{k-1}$.

Let

$$
M_{j}=\left(\begin{array}{ccccc}
A_{j} & B_{j+1} & 0 & \ldots & 0 \\
C_{j} & A_{j+1} & 0 & \ldots & 0 \\
& & \ddots & & \\
0 & \ldots & 0 & A_{k-2} & B_{k-1} \\
0 & \ldots & 0 & C_{k-2} & A_{k-1}
\end{array}\right),
$$

$j=1, \ldots, k-1$, and let $M_{k}=(1)$ and $M_{k+1}=(0)$. It follows by induction on $j$ that

$$
\operatorname{det}(M)=A_{1} \ldots A_{j}\left(c_{j} \operatorname{det}\left(M_{j+1}\right)-d c_{j-1} A_{j+1} \operatorname{det}\left(M_{j+2}\right)\right)
$$

for $j=1, \ldots, k-1$. By letting $j=k-1$ in (11), we have $\operatorname{det}(M)=$ $A_{1} \ldots A_{k-1} c_{k-1}$. Since $d=\frac{1}{4}\left(1-c / a^{2}\right)$ and $c / a^{2}>0$, either $d<0$ or $0<d<1 / 4$. If $d<0$ then $c_{j}>0$ for all $j$ by the definition of the sequence $c_{j}$. If $0<d<1 / 4$, let $b_{j}=c_{j} / c_{j-1}$ for all $j$ such that $c_{j-1} \neq 0$. It follows by induction on $j$ that $1 / 2<b_{j} \leq 1$. Hence $c_{j}>0$ for all $j$.

Lemma 3.3. Let $k$ be even and let $b_{3}, \ldots, b_{k} \in \mathbb{Q}$. Define $\sigma_{1}=1$ and

$$
\sigma_{j}=\sum_{3 \leq i_{1}<\ldots<i_{j-1} \leq k} b_{i_{1}} \ldots b_{i_{j-1}}, \quad j=2, \ldots, k-1 .
$$

Then it is impossible to have

$$
\sigma_{2 j}=0 \quad \text { and } \quad \sigma_{2 j+1}>0 \quad \text { for all } j=1, \ldots,(k-2) / 2 .
$$


Pr o of. Suppose (12) is true. First we show by induction on $j$ that

$$
\sigma_{k-3} \leq-b_{3}^{j} \sum_{4 \leq i_{1}<\ldots<i_{k-(j+4)} \leq k} b_{i_{1}} \ldots b_{i_{k-(j+4)}}-b_{3}^{-1} b_{4} \ldots b_{k},
$$

$j=2,4, \ldots, k-4$, where we treat a sum over the empty set as 1 . Since

$$
\begin{aligned}
\sigma_{j} & =\sum_{3 \leq i_{1}<\ldots<i_{j-1} \leq k} b_{i_{1}} \ldots b_{i_{j-1}} \\
& =b_{3} \sum_{4 \leq i_{1}<\ldots<i_{j-2} \leq k} b_{i_{1}} \ldots b_{i_{j-2}}+\sum_{4 \leq i_{1}<\ldots<i_{j-1} \leq k} b_{i_{1}} \ldots b_{i_{j-1}}
\end{aligned}
$$

and $\sigma_{2 j}=0$ and $\sigma_{2 j+1}>0$, we have

$$
\sum_{4 \leq i_{1}<\ldots<i_{2 j-1} \leq k} b_{i_{1}} \ldots b_{i_{2 j-1}}=-b_{3} \sum_{4 \leq i_{1}<\ldots<i_{2 j-2} \leq k} b_{i_{1}} \ldots b_{i_{2 j-2}}
$$

and

$$
-\sum_{4 \leq i_{1}<\ldots<i_{2 j} \leq k} b_{i_{1}} \ldots b_{i_{2 j}}<b_{3} \sum_{4 \leq i_{1}<\ldots<i_{2 j-1} \leq k} b_{i_{1}} \ldots b_{i_{2 j-1}},
$$

$j=1, \ldots,(k-2) / 2$. Hence,

$$
-\sum_{4 \leq i_{1}<\ldots<i_{2 j} \leq k} b_{i_{1}} \ldots b_{i_{2 j}}<-b_{3}^{2} \sum_{4 \leq i_{1}<\ldots<i_{2 j-2} \leq k} b_{i_{1}} \ldots b_{i_{2 j-2}} .
$$

By setting $j=(k-2) / 2$ in (14) we have $\sum_{4 \leq i_{1}<\ldots<i_{k-4} \leq k} b_{i_{1}} \ldots b_{i_{k-4}}=$ $-b_{3}^{-1} b_{4} \ldots b_{k}$, so that

$$
\sigma_{k-3}=-b_{3}^{2} \sum_{4 \leq i_{1}<\ldots<i_{k-6} \leq k} b_{i_{1}} \ldots b_{i_{k-6}}-b_{3}^{-1} b_{4} \ldots b_{k},
$$

which shows (13) for $j=2$.

Suppose that (13) is true for $j$. Then by the induction hypothesis and (15),

$$
\begin{aligned}
\sigma_{k-3} & \leq-b_{3}^{j} \sum_{4 \leq i_{1}<\ldots<i_{k-(j+4)} \leq k} b_{i_{1}} \ldots b_{i_{k-(j+4)}}-b_{3}^{-1} b_{4} \ldots b_{k} \\
& <-b_{3}^{j+2} \sum_{4 \leq i_{1}<\ldots<i_{k-(j+6)} \leq k} b_{i_{1}} \ldots b_{i_{k-(j+6)}}-b_{3}^{-1} b_{4} \ldots b_{k},
\end{aligned}
$$

which shows (13) for $j+2$. Hence (13) is proved.

Let $j=k-4$. Then

$$
\sigma_{k-3} \leq-\left(b_{3}^{k-4}+b_{3}^{-1} b_{4} \ldots b_{k}\right)<0
$$

since $k-4$ is even and $b_{3} \ldots b_{k}=\sigma_{k-1}>0$. However, this contradicts $\sigma_{k-3}>0$. Hence, (12) is impossible. 
4. Proof of Theorem 1.1. Let $l$ be the dimension of the vector space over $\mathbb{Q}$ spanned by $\alpha_{1}, \ldots, \alpha_{k}$. By possibly reordering the $\alpha_{i}$ 's, we may take $\alpha_{1}, \ldots, \alpha_{l}$ as basis of this vector space so that

$$
\alpha_{i}=\sum_{j=1}^{l} a_{i j} \alpha_{j}, \quad a_{i j} \in \mathbb{Q}, \quad i=l+1, \ldots, k .
$$

If $l=1$, then either $q(n) \sim c \gamma n^{k}, c \in \mathbb{Q}$, which is uniformly distributed $(\bmod 1)$ since $c \gamma n^{k}$ is a polynomial with irrational coefficient, or $q(n) \sim$ $c[\alpha n]^{k} \gamma, c \in \mathbb{Q}, \alpha$ irrational. Since $c[\alpha n]^{2} \gamma$ is uniformly distributed $(\bmod 1)$ by Proposition 1.2 and $q^{h}(n) \sim c k[\alpha n]^{k-1} \gamma$, it follows by induction and van der Corput's difference theorem that $q(n)=c[\alpha n]^{k} \gamma$ is uniformly distributed $(\bmod 1)$.

If $l=2$, then

$$
q(n) \sim\left[\alpha_{1} n\right]\left[\alpha_{2} n\right] \prod_{i=3}^{k}\left(a_{i 1}\left[\alpha_{1} n\right]+a_{i 2}\left[\alpha_{2} n\right]\right) \gamma=\sum_{i=1}^{k-1} a_{i}\left[\alpha_{1} n\right]^{k-i}\left[\alpha_{2} n\right]^{i} \gamma
$$

where

$$
\begin{gathered}
a_{1}=\prod_{i=3}^{k} a_{i 1}, \\
a_{2}=\sum_{i=3}^{k}\left(\prod_{j \neq i} a_{j 1}\right) a_{i 2}, \\
\vdots \\
a_{k-1}=\prod_{i=3}^{k} a_{i 2} .
\end{gathered}
$$

Note that since $q(n) \neq 0$ there exists $i, 1 \leq i \leq k-1$ so that $a_{i} \neq 0$.

Let $a_{0}=a_{k}=0$ so that we can write

$$
q(n) \sim \sum_{i=0}^{k} a_{i}\left[\alpha_{1} n\right]^{k-i}\left[\alpha_{2} n\right]^{i} \gamma
$$

By van der Corput's difference theorem, $q(n)$ is uniformly distributed $(\bmod 1)$ if

$$
\begin{gathered}
q^{h}(n) \sim\left[\alpha_{1} h\right]\left(\sum_{i=0}^{k} a_{i}(k-i)\left[\alpha_{1} n\right]^{k-i-1}\left[\alpha_{2} n\right]^{i} \gamma\right) \\
+\left[\alpha_{2} h\right]\left(\sum_{i=0}^{k} a_{i} i\left[\alpha_{1} n\right]^{k-i}\left[\alpha_{2} n\right]^{i-1} \gamma\right)
\end{gathered}
$$


is uniformly distributed $(\bmod 1)$, and by Lemma $2.5, q^{h}(n)$ is uniformly distributed $(\bmod 1)$ if either

or

$$
v_{1}(n)=\sum_{i=0}^{k-1} a_{i}(k-i)\left[\alpha_{1} n\right]^{k-i-1}\left[\alpha_{2} n\right]^{i} \gamma
$$

$$
v_{2}(n)=\sum_{i=1}^{k} a_{i} i\left[\alpha_{1} n\right]^{k-i}\left[\alpha_{2} n\right]^{i-1} \gamma
$$

is uniformly distributed $(\bmod 1)$. The same argument can be repeated for $v_{1}(n)$ and $v_{2}(n)$. Note that $v_{1}(n)$ and $v_{2}(n)$ can be seen as the partial derivatives of the polynomial function

$$
f(x, y)=\sum_{i=0}^{k} a_{i} x^{k-i} y^{i} \gamma
$$

on $\mathbb{R}^{2}$, evaluated at $\left(\left[\alpha_{1} n\right],\left[\alpha_{2} n\right]\right)$. So by using induction it follows from van der Corput's difference theorem and Lemma 2.5 that $q(n)$ is uniformly distributed $(\bmod 1)$ if at least one of the $(k-2)$ th partial derivatives evaluated at $\left(\left[\alpha_{1} n\right],\left[\alpha_{2} n\right]\right)$,

$$
Q_{j}(n)=\frac{\partial^{k-2} f}{\partial x^{k-2-j} \partial y^{j}}\left(\left[\alpha_{1} n\right],\left[\alpha_{2} n\right]\right), \quad j=0,1, \ldots, k-2,
$$

is uniformly distributed $(\bmod 1)$. Now,

so that

$$
\begin{aligned}
\frac{\partial^{k-2} f}{\partial x^{k-2-j} \partial y^{j}}(x, y)= & a_{j} \frac{(k-j) !}{2 !} j ! x^{2} \gamma+a_{j+1}(k-j-1) !(j+1) ! x y \gamma \\
& +a_{j+2}(k-j-2) ! \frac{(j+2) !}{2 !} y^{2} \gamma,
\end{aligned}
$$

$$
\begin{aligned}
Q_{j}(n)= & \frac{(k-j) !}{2 !} j ! a_{j}\left[\alpha_{1} n\right]^{2} \gamma+(k-j-1) !(j+1) ! a_{j+1}\left[\alpha_{1} n\right]\left[\alpha_{2} n\right] \gamma \\
& +(k-j-2) ! \frac{(j+2) !}{2 !} a_{j+2}\left[\alpha_{2} n\right]^{2} \gamma,
\end{aligned}
$$

$j=0, \ldots, k-2$. If none of the $Q_{j}(n)$ 's is uniformly distributed $(\bmod 1)$, by Proposition 3.1 there exist $a, c \in \mathbb{Q}, c>0, c \neq a^{2}$, such that

$$
\begin{aligned}
& \frac{(k-j) !}{2 !} j ! a_{j}+a(k-j-1) !(j+1) ! a_{j+1} \\
& \quad+\left(a^{2}-c\right)(k-j-2) ! \frac{(j+2) !}{2 !} a_{j+2}=0, \quad j=0, \ldots, k-2 .
\end{aligned}
$$

We will show that this leads to a contradiction. Now, use the fact that $a_{0}=a_{k}=0$, and let $a_{1}, \ldots, a_{k-1}$ be the unknowns in the system (17) of $k-1$ equations. 
If $a \neq 0$, the system (17) has the unique solution $(0, \ldots, 0)$ if the matrix

$$
M=\left(\begin{array}{cccccc}
A_{1} & B_{2} & 0 & 0 & \ldots & 0 \\
C_{1} & A_{2} & B_{3} & 0 & \ldots & 0 \\
0 & C_{2} & A_{3} & B_{4} & \ldots & 0 \\
& & & \ddots & & \\
0 & \ldots & 0 & C_{k-3} & A_{k-2} & B_{k-1} \\
0 & \ldots & 0 & 0 & C_{k-2} & A_{k-1}
\end{array}\right)
$$

is non-singular, where

$$
A_{j}=(k-j) ! j ! a, \quad B_{j}=(k-j) ! \frac{j !}{2 !}\left(a^{2}-c\right), \quad C_{j}=\frac{(k-j) !}{2 !} j ! .
$$

Since

$$
B_{j}=\frac{a^{2}-c}{2 a} A_{j} \quad \text { and } \quad C_{j}=\frac{1}{2 a} A_{j},
$$

it follows that $C_{j} B_{j+1}=d A_{j} A_{j+1}$, where $d=\frac{1}{4}\left(1-c / a^{2}\right)$. So by Lemma 3.2, $\operatorname{det}(M) \neq 0$. Therefore $q(n)=0$, a contradiction.

If $a=0$, then (17) gives

$$
\frac{(k-j) !}{2 !} j ! a_{j}=c(k-j-2) ! \frac{(j+2) !}{2 !} a_{j+2}, \quad j=0, \ldots, k-2 .
$$

Since $a_{0}=a_{k}=0$, we have $a_{2 j}=0$ for all $j$. If $k$ is odd, then we also have $a_{2 j+1}=0$ for all $j$ such that $q(n)=0$, a contradiction.

Let $k$ be even. If $a_{1}=0$ then $a_{i}=0$ for all $i$. So we may assume that $a_{1} \neq 0$. It follows from (18) that $a_{2 j+1} / a_{1}>0$ for all $j$. Recall that the $a_{i}$ 's satisfy the equations (16). Let $b_{i}=a_{i 2} / a_{i 1}$. Then it follows from (16) and (18) that

$$
\frac{a_{2 j}}{a_{1}}=\sum_{3 \leq i_{1}<\ldots<i_{2 j-1} \leq k} b_{i_{1}} \ldots b_{i_{2 j-1}}=0, \quad j=1, \ldots,(k-2) / 2
$$

and

$$
\frac{a_{2 j+1}}{a_{1}}=\sum_{3 \leq i_{1}<\ldots<i_{2 j} \leq k} b_{i_{1}} \ldots b_{i_{2 j}}>0, \quad j=0, \ldots,(k-2) / 2,
$$

which by Lemma 3.3 is impossible. This ends the proof for the case $l=2$.

Let $l>2$. We will show by induction on $k$ that

$$
q(n) \sim\left(\prod_{i=1}^{l}\left[\alpha_{i} n\right]\right) \prod_{i=l+1}^{k}\left(\sum_{j=1}^{l} a_{i j}\left[\alpha_{j} n\right]\right) \gamma
$$

is uniformly distributed $(\bmod 1)$ for any $l \leq k$.

If $k=3$ and $l=3$ then $\alpha_{1}, \alpha_{2}, \alpha_{3}$ are rationally independent and

$$
q^{h}(n) \sim\left[\alpha_{1} h\right]\left[\alpha_{2} n\right]\left[\alpha_{3} n\right] \gamma+\left[\alpha_{2} h\right]\left[\alpha_{1} n\right]\left[\alpha_{3} n\right] \gamma+\left[\alpha_{3} h\right]\left[\alpha_{1} n\right]\left[\alpha_{2} n\right] \gamma .
$$


By Lemma 2.5, it is enough that some $\left[\alpha_{i} n\right]\left[\alpha_{j} n\right] \gamma$ is uniformly distributed $(\bmod 1), i \neq j, i, j \in\{1,2,3\}$. If all of them fail to be uniformly distributed $(\bmod 1)$, then by Proposition 1.2,

$$
\frac{\alpha_{i}}{\alpha_{j}}=a_{i j} \sqrt{c} \text { and } \gamma=k_{0} \sqrt{c}, \quad i, j=1,2,3, a_{i j}, k_{0} \in \mathbb{Q} \backslash\{0\} .
$$

If this is the case then $\alpha_{1}=a_{12} \sqrt{c} \alpha_{2}=a_{13} \sqrt{c} \alpha_{3}$, which contradicts $\alpha_{1}, \alpha_{2}$, $\alpha_{3}$ being rationally independent. So $q(n)$ is uniformly distributed $(\bmod 1)$ if $k=3$.

Let $k>3$ and suppose $q(n)$ is uniformly distributed $(\bmod 1)$ if $\operatorname{deg}(q)<$ $k$. By rewriting the expressions (19) for $q(n)$, we have

$$
\begin{aligned}
q(n) \sim & \sum_{r=0}^{k-l}\left[\alpha_{1} n\right]^{k-l+1-r}\left(\prod_{i=2}^{l}\left[\alpha_{i} n\right]\right) \\
& \times \sum_{l<i_{1}<\ldots<i_{r} \leq k}\left(\prod_{j \neq i_{1}, \ldots, i_{r}} a_{j 1}\right) \prod_{j=1}^{r}\left(\sum_{s=2}^{l} a_{i_{j} s}\left[\alpha_{s} n\right]\right) .
\end{aligned}
$$

Let $l_{1}, \ldots, l_{m}, 0 \leq m \leq k-l$, be all the indices $i$ for which $a_{i 1}=0$. Then

$$
\begin{aligned}
q(n) \sim & \sum_{r=m}^{k-l}\left[\alpha_{1} n\right]^{k-l+1-r}\left(\prod_{i=2}^{l}\left[\alpha_{i} n\right]\right) \sum_{l<i_{1}<\ldots<i_{r} \leq k}\left(\prod_{j \neq i_{1}, \ldots, i_{r}} a_{j 1}\right) \\
& \times \prod_{j=1}^{r}\left(\sum_{s=2}^{l} a_{i_{j} s}\left[\alpha_{s} n\right]\right)
\end{aligned}
$$

and if

$$
q_{1}(n)=\left(\prod_{i=2}^{l}\left[\alpha_{i} n\right]\right) \sum_{l<i_{1}<\ldots<i_{m} \leq k}\left(\prod_{j \neq i_{1}, \ldots, i_{m}} a_{j 1}\right) \prod_{j=1}^{m}\left(\sum_{s=2}^{l} a_{i_{j} s}\left[\alpha_{s} n\right]\right),
$$

then

$$
q_{1}(n)=\left(\prod_{i=2}^{l}\left[\alpha_{i} n\right]\right)\left(\prod_{j \neq l_{1}, \ldots, l_{m}} a_{j 1}\right) \prod_{j=1}^{m}\left(\sum_{s=2}^{l} a_{l_{j} s}\left[\alpha_{s} n\right]\right),
$$

which is a non-zero generalized polynomial of the form $\left(\prod_{i=1}^{k_{1}}\left[\lambda_{i} n\right]\right) \gamma$ where $k_{1}=m+l-1 \leq k-l+l-1=k-1$ and $l_{1}=l-1$. By the induction hypothesis, $q_{1}(n)$ is uniformly distributed $(\bmod 1)$ if $m \neq 0$ or $m=0$ and $l>3$.

If we see $q(n)$ as a polynomial function $f$ on $\mathbb{R}^{l}$ evaluated at $\left(\left[\alpha_{1} n\right],\left[\alpha_{2} n\right]\right.$, $\left.\ldots,\left[\alpha_{l} n\right]\right)$, we deduce similarly to the case $l=2$ that $q(n)$ is uniformly distributed $(\bmod 1)$ if one of the generalized polynomials

$$
\frac{\partial^{i} f}{\partial x_{1}^{i_{1}} \ldots \partial x_{l}^{i_{l}}}\left(\left[\alpha_{1} n\right],\left[\alpha_{2} n\right], \ldots,\left[\alpha_{l} n\right]\right), \quad i_{1}+\ldots+i_{l}=i, i \leq k-1,
$$


is uniformly distributed $(\bmod 1)$, and especially if

$$
\frac{\partial^{k-l+1-m} f}{\partial x_{1}^{k-l+1-m}}\left(\left[\alpha_{1} n\right],\left[\alpha_{2} n\right], \ldots,\left[\alpha_{l} n\right]\right)
$$

is uniformly distributed $(\bmod 1)$. From $(20)$ we see that

$$
\frac{\partial^{k-l+1-m} f}{\partial x_{1}^{k-l+1-m}}\left(\left[\alpha_{1} n\right],\left[\alpha_{2} n\right], \ldots,\left[\alpha_{l} n\right]\right)=(k-l+1-m) ! q_{1}(n),
$$

which we have shown is uniformly distributed $(\bmod 1)$ if $m \neq 0$ or $m=0$ and $l>3$. Note that if $m=0$ and $l=3$, then $(k-2) ! q_{1}(n)=a\left[\alpha_{1} n\right]\left[\alpha_{2} n\right] \gamma, a \in$ $\mathbb{Q}$, which may fail to be uniformly distributed $(\bmod 1)$. Since there was nothing special about $\alpha_{1}$, we could use $\alpha_{2}$ or $\alpha_{3}$ instead of $\alpha_{1}$. If the corresponding $m_{2} \neq 0$ or $m_{3} \neq 0$, then $q(n)$ is uniformly distributed $(\bmod 1)$ as above. However, if $m_{1}=m_{2}=m_{3}=0$, then the problem is reduced to showing that one of $\left[\alpha_{1} n\right]\left[\alpha_{2} n\right] \gamma,\left[\alpha_{2} n\right]\left[\alpha_{3} n\right] \gamma$ or $\left[\alpha_{1} n\right]\left[\alpha_{3} n\right] \gamma$ is uniformly distributed $(\bmod 1)$, which was proved under the case $k=l=3$. This completes the proof that $q(n)$ is uniformly distributed $(\bmod 1)$.

\section{References}

[1] J. Brezin, Applications of nilmanifold theory to diophantine approximations, Proc. Amer. Math. Soc. 33 (1972), 543-547.

[2] J. G. van der Corput, Diophantische Ungleichungen I. Zur Gleichverteilung modulo Eins, Acta Math. 56 (1931), 373-456.

[3] I. J. Håland, Uniform distribution of generalized polynomials, J. Number Theory 45 (1993), 327-366.

[4] L. Kuipers and H. Niederreiter, Uniform Distribution of Sequences, Wiley, 1974.

[5] Y. Peres, Application of Banach limits to the study of sets of integers, Israel J. Math. 62 (1988), 17-31.

[6] H. Weyl, Über die Gleichverteilung von Zahlen mod. Eins, Math. Ann. 77 (1916), 313-352.

AGDER COLLEGE OF ENGINEERING

N-4890 GRIMSTAD

NORWAY

Received on 29.3.1993

and in revised form on 13.9.1993 